\title{
Gestaltung der Arbeit mit Kollaborationsplattformen
}

\section{Ergebnisse aus dem Verbundvorhaben CollaboTeam}

Thomas Hardwig, Stefan Klötzer, Alfred Mönch, Tobias Reißmann,
Carsten Schulz und Marliese Weißmann

\subsection{Betriebliche Entwicklung und Erprobung mit wissenschaftlicher Begleitung}

In den Unternehmen verbreiten sich in den letzten Jahren zunehmend Kollaborationsplattformen, auf denen verschiedene Anwendungen für die unternehmensweite Kommunikation und das Wissensmanagement integriert werden, um eine Zusammenarbeit unabhängig von Ort und Zeit $\mathrm{zu}$ ermöglichen [1]. Kollaborationsplattformen schaffen einen virtuellen Ort, an dem Mitglieder eines Teams oder eines Projektes zusammenarbeiten. Sie bieten zudem einen unternehmensweiten Zugriff auf Inhalte sowie die selbstgesteuerte Bildung von virtuellen Gruppen („Communities“) zu bestimmten Fragen oder Aufgaben. Auch die Zusammenarbeit mit Kunden und anderen Externen kann damit unterstützt werden.

Die hauptsächlichen Kosten und Risiken für die Nutzung solch neuer, digitaler Technologien liegen nicht bei der Anschaffung, sondern bei ihrer Integration in die

T. Hardwig $(\bowtie) \cdot$ S. Klötzer

Georg-August-Universität Göttingen, Kooperationsstelle Hochschulen und Gewerkschaften, Göttingen, Deutschland

A. Mönch

Zeiss Digital Innovation GmbH, Dresden, Deutschland

T. Reißmann

Xenon Automatisierungstechnik GmbH, Dresden, Deutschland

C. Schulz

GIS Gesellschaft für InformationsSysteme AG, Hamburg, Deutschland

M. Weißmann

Soziologisches Forschungsinstitut Göttingen, Göttingen, Deutschland

(C) Der/die Autor(en) 2021

W. Bauer et al. (Hrsg.), Arbeit in der digitalisierten Welt,

https://doi.org/10.1007/978-3-662-62215-5_8 
Arbeitsabläufe und Prozesse eines Unternehmens. Zudem ist es Stand des Wissens, dass eine erfolgversprechende Nutzung digitaler Technologien in Unternehmen nur durch eine ganzheitliche, sozio-technischen Systemgestaltung zu erreichen ist [2, 3]. Dennoch überschätzen die Menschen, die in den Unternehmen Entscheidungen treffen, immer noch den unmittelbaren Nutzen einer Bereitstellung neuer, digitaler Technologien. Zugleich unterschätzen sie den Aufwand an Arbeitsgestaltung, der geleistet werden muss, damit die Potenziale der Technologien am Ende realisiert werden können. Dies gilt in besonderer Weise für kollaborative Anwendungen, möglicher Weise, weil deren Funktionalitäten vielfach aus dem privaten Gebrauch (z. B. Whatsapp usw.) vertraut sind. Zwar wird oftmals noch erkannt, dass für ihren erfolgreichen Einsatz im Unternehmen erhebliche Voraussetzungen zu schaffen sind [3], doch dass ihr konsequenter Einsatz durchaus auch disruptive Veränderungen der Kommunikation und Zusammenarbeit im Unternehmen bewirken kann, haben die wenigsten im Blick [4]. Aufgrund einer fehlenden Aufbereitung des vorhandenen Wissens und vorhandener Forschungsdefizite zum Thema Kollaborationsplattformen, erhalten Verantwortliche für die Arbeitsgestaltung bislang zu wenig Unterstützung für die betriebliche Bewältigung der digitalen Transformation ihres Unternehmens.

Vor diesem Hintergrund war das Gesamtziel des Verbundprojekts, gemeinsam wissenschaftlich fundierte Konzepte zur Arbeitsgestaltung sowie zur nachhaltigen Personal- und Organisationsentwicklung für die Nutzung von Kollaborationsplattformen im Rahmen von Team- und Projektarbeit zu erarbeiten und umzusetzen. Die soziotechnischen Gestaltungskonzepte sollten sowohl den Kriterien humanorientierter Arbeit als auch betriebswirtschaftlichen Anforderungen genügen. Sie sollten zudem die Fähigkeit der Betriebe zur Reaktion auf sich wandelnde Kundenanforderungen und Umweltbedingungen fördern, indem die Möglichkeiten, die die Digitalisierung bietet, ausgeschöpft werden und damit die interne und betriebsübergreifende Kooperations- und Innovationsfähigkeit verbessert wird. Um dieses Ziel zu erreichen, haben die Projektpartner gemeinsam drei Arbeitsschritte realisiert.

1. Es erfolgte eine Entwicklung und Erprobung betrieblicher Lösungen zur Arbeitsgestaltung in den drei Partnerunternehmen. Nach einer wissenschaftlichen Bestandsaufnahme wurden in jedem Unternehmen in zwei Pilotphasen Gestaltungsansätze erprobt, ihre Ergebnisse reflektiert und weiterentwickelt. Während in der ersten Pilotphase eher die interne Zusammenarbeit im Mittelpunkt stand, wurde in der zweiten die Zusammenarbeit mit Kunden betrachtet. Zudem wurde in dieser Phase in allen drei Unternehmen das Produkt MS Teams erprobt. Jedes Unternehmen formulierte am Ende des Entwicklungsprozesses eine Roadmap für den weiteren Einsatz von Kollaborationsplattformen.

2. Es wurde ein Gestaltungsmodell für den Einsatz von Kollaborationsplattformen in Unternehmen sowie Empfehlungen für die Arbeit mit Kollaborationsplattformen entwickelt. Deren Grundlagen bilden die Entwicklung von Gestaltungslösungen in den drei Partnerunternehmen, die Ergebnisse aus der Bestandsaufnahme und den Intensiv- 
fallstudien (Interviews und Gruppengespräche mit Nutzerinnen und Nutzern sowie mit Verantwortlichen für die Arbeitsgestaltung) der wissenschaftlichen Begleitung, eine Bestandsaufnahme der Nutzungserfahrungen von $101 \mathrm{KMU}$ in Niedersachsen und Sachsen [5, 6] sowie einer Auswertung der wissenschaftlichen Literatur.

3. Parallel zu diesen Aktivitäten erfolgte der Aufbau eines Netzwerkes zum Austausch der gemachten Erfahrungen und Erkenntnisse sowohl über selbst organisierte Fachtagungen, Dialogveranstaltungen und Workshops für Betriebs- und Personalräte, als auch durch die Teilnahme an praxisorientierten Veranstaltungen sowie wissenschaftlichen Tagungen im nationalen und internationalen Rahmen. Zur Unterstützung der Netzwerkaktivitäten wurden die Webseite collaboteam.de mit aktuellen Informationen sowie verschiedene Publikationen erstellt.

\subsection{Gestaltung der Arbeit mit Kollaborationsplattformen als Beitrag zur humanverträglichen Digitalisierung der Arbeit}

Forschungsergebnisse zu kollaborativen Anwendungen sind nicht leicht $\mathrm{zu}$ finden. Der Gegenstand wird von unterschiedlichen Disziplinen unter mindestens zwölf verschiedenen Begriffen behandelt und es liegen erst wenige Forschungsergebnisse vor [7]. Insofern füllt das Projekt CollaboTeam eine Forschungslücke und stellt Wissen bereit, das in der betrieblichen Praxis dringend gebraucht wird.

Wir verwenden den Begriff der kollaborativen Anwendungen, bevorzugen aber inzwischen den Begriff der Kollaborationsplattform aus zwei Gründen: Es wird mit dem Kollaborationsbegriff etwa im Unterschied zur Teamplattform bewusst offengehalten, auf welche sozialen Einheiten (Personen, Gruppe bis hin zur gesamten Organisation) sich die Kollaboration bezieht. Zweitens versinnbildlicht der Begriff Plattform einen Ort, an dem ein Teil der Arbeit erledigt wird und Möglichkeiten der Selbstorganisation, Kommunikation und sozialen Vernetzung bestehen. Damit ist angesprochen, dass in der Regel verschiedene Anwendungen technisch in einer Kollaborationsplattform integriert werden, also ein digitaler Arbeitsplatz mit wenig Schnittstellen geschaffen werden kann. Dabei können die Funktionalitäten für einzelne Nutzergruppen innerhalb eines Unternehmens sehr differenziert angeboten werden.

Die Präsentation wesentlicher Ergebnisse des Verbundvorhabens CollaboTeam konzentriert sich - aufgrund des begrenzten Rahmens dieser Publikation - auf ein Gestaltungskonzept für die Arbeit mit Kollaborationsplattformen. Es wurde in enger Zusammenarbeit mit den Partnerunternehmen entwickelt und soll betrieblichen Akteuren in der Praxis Orientierung zur Einführung und Nutzung von Kollaborationsplattformen geben. Im Weiteren stellen wir das Gestaltungsmodell vor und unterlegen es mit Erfahrungen aus den Partnerunternehmen. Darüber hinaus finden sich Ergebnisse des Projektverbundes CollaboTeam in weiteren Publikationen (siehe www.collaboteam.de). 


\subsubsection{Ein Gestaltungsmodell für die Arbeit mit Kollaborationsplattformen}

Die im Rahmen von CollaboTeam durchgeführten Fallstudien führen vor Augen, dass Kollaborationsplattformen (wie etwa MS Teams von Microsoft) ein hohes Potenzial für die soziale Vernetzung und Zusammenarbeit und Kommunikation in verschiedenen Formen in Unternehmen haben. Da die Plattformen nur dann genutzt werden und ihr Potenzial entfalten können, wenn sich ihre Nutzung nach den arbeitsbezogenen Bedürfnissen der Nutzerinnen und Nutzer richtet und Sinn für die Verrichtung ihrer Arbeit ergibt, ist ihr Einsatz prinzipiell herausfordernd und gestaltungsbedürftig.

Das Gestaltungsmodell soll Verantwortlichen für die Arbeitsgestaltung einen Orientierungsrahmen bieten, wie der Einsatz und die Nutzung von Kollaborationsplattformen in Organisationen menschenorientiert und effektiv gestaltet werden kann. Dabei folgt die Gestaltung dem sozio-technischen Grundprinzip der ,joint optimization“ [8] also dem Grundgedanken, dass Technologieeinsatz und soziale Organisation in enger Wechselbeziehung zu gestalten sind. Bei dem Gestaltungsmodell handelt es sich um eine aufgrund der Verbunderfahrungen weiterentwickelte Fassung des ersten Entwurfs [9].

Das Modell ist auf der Ebene eines konkreten Arbeitssystems anzuwenden. Doch zunächst ist es auf der ersten Ebene des Modells, der Strategie, sinnvoll, Ziele nicht nur bezüglich eines Arbeitssystems, sondern für ein System aus verschiedenen Arbeitssystemen zu formulieren. Analyse und Gestaltung - die nächsten beiden Ebenen - beziehen sich dann jeweils auf ein Arbeitssystem, wobei das Zusammenspiel der beteiligten sozialen und technischen Systeme (=Arbeitssystem) in den Mittelpunkt gestellt wird. Empfohlen wird den Beteiligten an der Arbeitsgestaltung ein Vorgehen in drei Schritten:

1. Strategie: Durch die Formulierung einer Roadmap für die Kollaboration werden Ziele für die Arbeitsgestaltung entwickelt und ein Rahmen für die weitere Arbeit mit Kollaborationsplattformen festgelegt.

2. Analyse: Hier wird der Gestaltungsbedarf in einem Arbeitssystem durch Analyse der Passung der Aufgabe, der eingesetzten Technologien und der beteiligten Nutzerinnen und ermittelt.

3. Gestaltung: Auf Grundlage der Analyseergebnisse wird der Gestaltungsbedarf abgeleitet und Maßnahmen für sechs Gestaltungsfelder des Modells entwickelt. Die Handlungsfelder haben sich - das wissen wir aufgrund vorliegender sowie eigener Forschungsergebnisse - für die Umsetzung von Gestaltungsmaßnahmen als relevante Stellschrauben für die Optimierung der Arbeit mit Kollaborationsplattformen erwiesen.

Die drei Schritte können zwar sequenziell durchlaufen werden, da sie aber in einem engen Wechselverhältnis stehen, ist zu erwarten, dass es rekursive Schleifen geben 
wird. Beispielsweise werden Analyseergebnisse dazu führen können, die Strategie zu modifizieren, und erfolgreich realisierte Maßnahmen schlagen sich in zukünftigen Analysen nieder. Im Folgenden werden die einzelnen Modell-Ebenen kurz beschrieben und wesentliche Ergebnisse aus den Partnerunternehmen dazu vorgestellt (Abb. 8.1).

\section{Strategie: Roadmap für die Kollaboration}

Der Einsatz von Kollaborationsplattformen in einem Unternehmen erfolgt auf der Grundlage der spezifischen Markt- und Produktionsanforderungen und mit Blick auf spezifische Aufgaben, die von den Beschäftigten erfüllt werden sollen, um den Wettbewerbsanforderungen genügen $\mathrm{zu}$ können. Daraus resultieren betriebsindividuell verschiedene Ziele, die durch den Einsatz erreicht werden sollen, wie die folgenden Beispiele aus den Partnerunternehmen zeigen:

XENON ist einer der führenden deutschen Hersteller von Automationsanlagen zur Montage und Prüfung von mechatronischen Bauteilen für die Branchen Automotive, Elektronik und Medizintechnik. Als unabhängiger Systemintegrator liefert XENON modulare High-Tech Fertigungslinien an Kunden weltweit. Innerhalb der XENON Unternehmensgruppe mit Werken in Deutschland, China und Mexiko müssen die verteilten Projektteams täglich gemeinsam an der Entwicklung und dem Bau der Automationsanlagen zusammenarbeiten. Dabei soll das langjährige Know-how des Stammsitzes in Dresden mit den lokalen Teams geteilt werden und eventuelle Probleme

\section{Strategie: Roadmap für die Kollaboration}
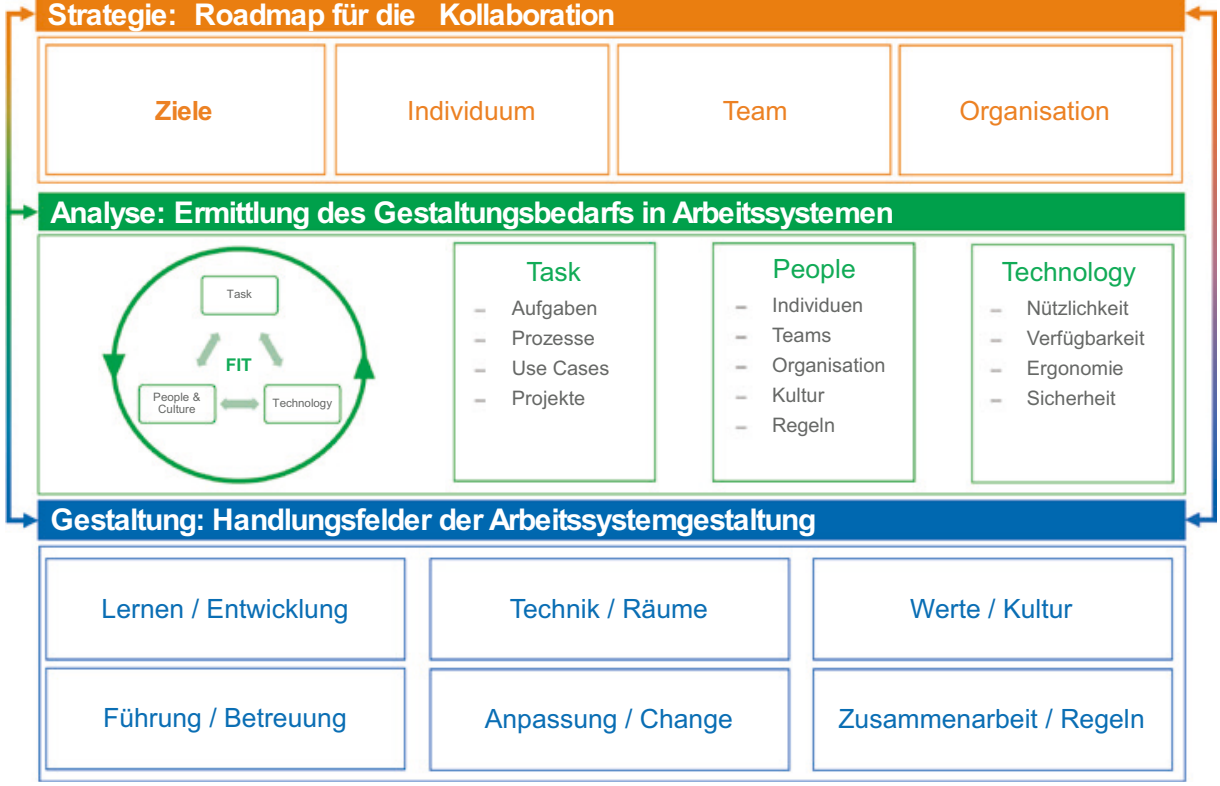

Abb. 8.1 Gestaltungsmodell für die Arbeit mit Kollaborationsplattformen (GMAK) 
in den Abläufen schnell innerhalb von einem Tag gelöst werden. Die vielfältigen Kommunikationsmöglichkeiten von Office 365 sollen intensiv genutzt werden, von der cloudbasierten Dateiablage, über Online-Notizbücher bis zur MS Teams Unterhaltung bzw. dem privaten Chat. Kollaboration kann somit quasi in Echtzeit über 3 Kontinente hinweg möglich werden. Für die Digitalisierung der internen Workflows hat sich XENON vorgenommen, eine eigene Applikation zur Prozessautomation zu entwickeln. Über digitale Formulare und ein dazugehöriges Aufgabenmanagement soll immer häufiger auf Papierdurchläufe verzichtet werden. Die Effizienz der Projektarbeit in der Entwicklung und in der Produktion soll damit deutlich gesteigert werden.

Die GIS AG, als einer der führenden Beratungsdienstleister im Umfeld New Work und Digital Workplace, unterstützt ihre Kunden im Rahmen der digitalen Transformation. Die GIS AG beschäftigt sich seit nunmehr zwanzig Jahren mit dem Thema Collaboration und Information Management und konzipiert seit über zehn Jahren gemeinsam mit ihren Kunden Social Intranets und Digital Workplace-Lösungen. Durch eine Verschiebung des Marktes im Umfeld des digitalen Arbeitsplatzes wurde das GIS Angebotsportfolio stärker diesem Markttrend angepasst. Die internen genutzten Werkzeuge sollten nun ebenfalls das neue Portfolio widerspiegeln und damit auch wesentlich ältere IT-Werkzeuge ablösen. Auch wenn schon Social Collaboration Systeme (Kollaborationsplattformen) im Einsatz sind, sollen die neuen Möglichkeiten einen weiteren Schub zur effizienten Projekt- und Teamarbeit ermöglichen. Dies wird vor allem im Hinblick auf die immer stärkere verteilte Projektarbeit im agilen Kontext gesehen.

Die Carl Zeiss Digital Innovation GmbH (ehem. Saxonia Systems AG) entwickelt individuelle Softwarelösungen für ihre Kunden innerhalb der Carl Zeiss AG und zahlreiche Geschäftspartner weiterer Branchen. Dabei verbindet sie State-of-the-Art Technologien, agiles Methoden Know-how und eine ausgeprägte Dienstleistungsmentalität zu neuen digitalen Produkten für ihre Auftraggeber. Die Carl Zeiss Digital Innovation beschäftigt Mitarbeiter deutschlandweit verteilt an sechs Standorten. Diese arbeiten in hochspezialisierten Teams, eigenverantwortlich und standortübergreifend zusammen, um die Herausforderungen ihrer komplexen Softwareentwicklungsprojekte zu meistern. Dazu nutzen die verteilten Teams meist die Konzepte agiler Vorgehensmodelle. Beide Faktoren, die Verfügbarkeit und Einbindung von Spezialwissen, sowie die Verwendung agiler Methoden erfordern ein hohes Maß an Transparenz, einen permanenten Wissensbzw. Informationsaustausch und kontinuierliche Abstimmungen zwischen den verteilten Mitgliedern dieser Teams. Die Basis dafür schaffen die Prinzipien und Methoden guter Kollaboration, sowie der richtige Einsatz moderner Kollaborationswerkzeuge gemeinsam bilden sie den digitalen Arbeitsplatz. Die ganzheitliche Einbettung dieses digitalen Arbeitsplatzes in die Unternehmensorganisation ist dabei essenziell für die Arbeit der Teams in ihren Projekten und somit den gesamten Erfolg der Carl Zeiss Digital Innovation. Es ist daher eine Hauptaufgabe des Unternehmens, diesen kontinuierlich weiterzuentwickeln - eigenständig und als Teil größerer Partnernetzwerke. 


\section{Analyse: Ermittlung des Gestaltungsbedarfs in Arbeitssystemen}

Um Gestaltungsaktivitäten ableiten zu können, wird die Passung zwischen „People“, „Technologie“ und „Task“ anhand der gesetzten Ziele überprüft. Dies ist in zweierlei Hinsicht wichtig, denn der Einsatz der Kollaborationsplattform soll einerseits zur effektiven Aufgaben- / Projektbearbeitung, und andererseits zur Erreichung der strategischen Ziele beitragen. Bei einem schlechten Fit müssen Gestaltungsmaßnahmen getroffen werden, die auf den Feldern der Ebene Gestaltung verortet sind.

- Fit „Task - Technology“: Die Aufgabe, der Prozess oder ein Projekt bilden den Ausgangspunkt für die Analyse. Ein Task kann sowohl eine einzelne Arbeitsaufgabe, ein Use-Case bzw. Prozess, der aus mehreren Aufgaben besteht oder ein Projekt als „größte“ Betrachtungseinheit sein. Überprüft wird, inwieweit der Einsatz der Technik die Aufgabenerfüllung erschwert oder unterstützt. Wie werden die Verfügbarkeit der Technik sowie die Sicherheit bewertet?

- Fit „Task - People“: Durch die technische Unterstützung ergeben sich Änderungen in der Art und Weise der Aufgabenerfüllung. Gibt es einen guten Fit zwischen der Aufgabe und den Menschen? Können die Menschen die Aufgabe problemlos erfüllen?

- Fit „People -Technology“: Wie ist die Kompetenz im Umgang mit der Kollaborationsplattform, die Teamkompetenz zur Selbststeuerung und inwieweit wirkt sich die Kultur der Zusammenarbeit oder des Unternehmens förderlich oder hinderlich auf das entstehende Arbeitssystem aus?

Zwei Beispiele aus den Partnerunternehmen skizzieren Erkenntnisse aus der Analyse:

Die GIS AG hat zu Beginn mit der Unterstützung der wissenschaftlichen Begleitung eine initiale Befragung durchgeführt, um zu erkennen, wo sie zu Beginn des Projektes steht und wo besonderes Verbesserungspotenzial vorhanden ist. Durch die mehrjährige Erfahrung mit einer älteren Kollaborationsplattform konnte sich bereits eine Kollaborationskultur entwickeln. Nutzerinnen und Nutzer äußerten keine Befürchtungen offen und transparent zu kommunizieren und die Plattform wurde bereits in der gesamten Belegschaft eingesetzt. Auffallend war ein Nutzungsabfall in den Betriebssupport Einheiten gegenüber den anderen Gruppen. Außerdem wurden zu dem Zeitpunkt noch nicht alle Möglichkeiten (Videotelefonie, Einbindung von Externen, etc.) ausgeschöpft. Mit der bisherigen Eigenentwicklung hat die GIS interne Prozesse sehr spezifisch abgedeckt, Usability und Userinterface sind jedoch inzwischen in die Jahre gekommen. Hier wurde deutlich, dass eine Umstellung auf ein moderneres System, was aber (im ersten Schritt) nicht denselben Integrationsgrad liefert, auf Widerstand stoßen wird. Auf diese Aspekte wurde in den kommenden Piloten besonderes Augenmerk gelegt.

Die Carl Zeiss Digital Innovation hat zur Verbesserung ihrer Kollaborationslösung für agile verteilte Zusammenarbeit für die Analyse des Status quo einerseits eine unternehmensweite Umfrage zum Konzept für verteilte agile Zusammenarbeit, andererseits von diesem Fokus losgelöste Team-Audits durchgeführt. Letztere gaben Aufschluss 
über die Zusammensetzung des Teams und der Art der Zusammenarbeit (,people“). Die unternehmensweite Umfrage zielte konkreter darauf ab, die Bekanntheit des eigenen Konzepts sowie konkrete Schmerz- und Verbesserungspunkte zu analysieren.

Das Ergebnis der Analyse zeigte, dass für durchweg technisch versierte Teammitglieder der Umgang mit den Kollaborationswerkzeugen prinzipiell kein Problem darstellt. Mitunter kommt es allerdings dazu, dass aufgrund ungünstiger einzelner Parameter im Setup die verteilte agile Zusammenarbeit nicht optimal funktioniert. Diese Aussage gilt einerseits für die Ausgestaltung des Raums und der Werkzeuge, andererseits darüber hinaus für die Implementierung der Rollen, Prozesse und der Teamdynamik selbst.

\section{Gestaltung: Handlungsfelder der Arbeitssystemgestaltung}

Der während der Analyse identifizierte Handlungsbedarf soll dann in der Gestaltungsphase in praktische Maßnahmen übersetzt und realisiert werden. Die Handlungsfelder werden kurz angesprochen und mit Beispielen aus der betrieblichen Umsetzung illustriert.

Technik/Räume Gestaltung der Technik hinsichtlich Nutzen für die Arbeit der Zielgruppen, Nutzerfreundlichkeit ihrer Bedienung, Ergonomie und Datensicherheit. Sowohl virtuelle Räume als auch physische Räume werden an die Bedürfnisse der NutzerInnen und im Hinblick auf die Anforderungen aus der Aufgabe (z. B. Anforderungen an die Zusammenarbeit zwischen bestimmten Stakeholdern) ausgerichtet.

Technik ist nicht alles - aber ohne sie funktioniert verteilte agile Entwicklung nicht. Ziel für die Carl Zeiss Digital Innovation GmbH ist es also, die technischen Hilfsmittel so zu gestalten, dass sie in jeder Hinsicht einfach anzuwenden sind und so zu gestalten, dass sie die Anwenderinnen und Anwender bestmöglich in ihrer Zusammenarbeit unterstützt und nicht beispielsweise durch Medienbrüche oder komplexe Bedienung (zusätzlich) behindert. Bereits im ursprünglichen Konzept wurden Empfehlungen für den optimalen verteilten Teamraum gegeben, doch bei der Umsetzung wurde festgestellt, dass die realen Gegebenheiten oft zu Kompromissen zwingen. Es ist also nötig zu analysieren, welche Setups eine brauchbare Alternative darstellen und welche einen solchen Bruch erzeugen, dass sie das Gesamtkonzept behindern.

Führung/Betreuung Festlegung der Aufgaben von Führungsrollen zur Unterstützung der Zusammenarbeit und neuer Funktionen und Rollen zur Führung der Teams, Projekte oder Abteilungen. Definition von Betreuungsrollen, die für Nutzerschaft ansprechbar sind oder aktiv das Funktionieren und Verbessern der technischen und sozialen Systeme, etwa die Einhaltung der Nutzungsregeln beinhalten.

Das von der Carl Zeiss Digital Innovation GmbH favorisierte Framework Scrum zur verteilten agilen Entwicklung unterstützt nicht nur effektiv in der Identifizierung und Umsetzung kontinuierlichen Verbesserungspotenzials, es bietet auch die prädestinierte Rolle zur begleitenden Führung der Teams: den Scrum Master. Er oder sie coacht das Team auf dem Weg der stetigen kontinuierlichen Verbesserung und lässt diesbezüg- 
lich die Grenzen zwischen Team und Organisation verschwimmen: Wie kann die Organisation die optimale Arbeit der Teams unterstützen, die dies ihrerseits durch die erfolgreiche Mitwirkung an der Erreichung der Unternehmensziele danken? Ein Beispiel auf diesen Weg war die Gestaltung von Teamräumen, welche oftmals zu klein für die vielen Teammitglieder und wenig optimiert für verteiltes Arbeiten waren. Durch eine umfangreiche Umbaumaßnahme konnten dadurch einige neue, besser auf die Bedürfnisse der verteilten Teams ausgerichtete Räume entstehen.

Lernen/Entwicklung Weiterentwicklung von individuellen und teambezogenen Kompetenzen zur Nutzung von Kollaborationsplattformen und zur Veränderung der Zusammenarbeit z. B. im Rahmen von Onboarding, E-Learning Maßnahmen, Schulungen, Trainings.

Innerhalb des GIS Projektes wurden zwei Piloten durchgeführt, die unterschiedliche Anforderungen hatten. Zunächst sollte das bestehende E-Mail und das Chat- und WebMeeting System ersetzt werden (kommunikationsgetriebene Arbeit). Hierbei wurden die Altsysteme abgeschaltet, sodass ein alternatives Zurückgreifen auf alte Werkzeuge nicht möglich war. Da diese Systeme aber nur geringe Auswirkungen auf eine Verhaltensänderung haben (mail wird weiterhin genutzt), wurde die Hürde bei der Nutzung eher beim Umgang mit dem neuen System selbst und der Motivation zum Ausprobieren gesehen. Es wurde bewusst auf klassische Klassenraum-Trainings verzichtet, sondern nur per Web Meeting eine kurze Einführung der wichtigsten Anwendungsfälle und die damit verbundene Umstellung mit dem neuen System erläutert. Ein weiterer wichtiger Baustein im Lernprozess war, mögliche positive Effekte des neuen Systems ebenfalls in kurzen Abständen zu kommunizieren, damit die Nutzer mit einer positiven Einstellung neue Lernerfahrungen anstreben. Parallel wurde ein Online Forum für Fragen und Diskussionen initiiert, welches auch bei anderen vorherigen Projekten schon erfolgreich eingesetzt wurde. Durch die Kombination konnten in kürzester Zeit alle Mitarbeiter erfolgreich umgestellt werden.

Im zweiten Piloten wurde dann die Social Collaboration Plattform ausgewechselt (kollaborative Arbeit), zunächst beschränkt für den Anwendungsfall der Projektarbeit. Dazu wurden wie im ersten Piloten genauso befähigende Maßnahmen durchgeführt. Es wurde aber recht schnell klar, dass obwohl diese Art der Zusammenarbeit bekannt war, doch weitere Regeln und Kommunikation benötigt wurden, um den kollaborativen Prozess weiter zu optimieren. Hierzu wurden Umfragen gestartet, um Fehlstellungen zu erkennen und bei Bedarf weitere Regelwerke zu etablieren oder Informationen zu verteilen. Es wurde aber weiterhin darauf verzichtet tiefergehende Schulung im Klassenraum anzubieten. Vielmehr wurden kurze Schulungen oder „Tipps \& Tricks“ per Web Session angeboten. Zu Beginn des Projektes wurden nach dem „Floorwalker“-Prinzip eine offene Sprechstunde vom Projektteam angeboten, um neue Anwendungsfälle zu diskutieren. Als Fazit kann die GIS für sich ziehen, dass auch ohne große Trainingsprogramme heute die Werkzeuge flächendeckend im Einsatz sind. Dabei wurde die 
Projektarbeit sehr schnell in weitere Anwendungsfälle übertragen, nachdem eine gewisse Medienkompetenz mit dem neuen System aufgebaut werden konnte.

Anpassung/Change Maßnahmen im Rahmen der Einführung und Nutzung oder Weiterentwicklung der Zusammenarbeit mit Kollaborationsplattformen.

Die Besonderheit der Modernisierung bei der GIS ist darin zu sehen, dass die bisherigen Werkzeuge schon längere Zeit im Einsatz waren und über die Jahre sehr auf die Anforderungen der GIS angepasst worden sind. Viele Prozesse wurden durchgängig in diversen Anwendungen integriert abgebildet. Daher wurden einige Prozesse (Angebot und Abrechnung und Vertriebsprozesse) zunächst ausgeklammert, um sich auf die Teamund Projektarbeit zu fokussieren, auch im Wissen, dass hier zunächst noch ein Medienbruch in der Bearbeitung existiert.

Trotz einer besseren Optik und Usability der neuen Werkzeuge, war die unternehmensweite Nutzung von daher kein Selbstläufer. Im zweiten Piloten wurden besonders die Kommunikation und der Dialog mit den Mitarbeitern noch weiter und frühzeitiger verstärkt, um allen Beteiligten auch zu erläutern, dass zunächst noch Zwischenlösungen existieren werden. Innerhalb des Dialogs kam dann der Wunsch aus der Belegschaft auf, zunächst nur mit der Team- und Projektarbeit zu starten und die tiefen, integrierten Prozesse später zu überführen. Dieser Impuls wurde aufgegriffen. Gerade der frühzeitige Dialog und das Mitgestalten der eigentlichen Umstellung, die den eigenen Arbeitsplatz wesentlich beeinflusst, hat bei den Mitarbeitern eine positive Resonanz gegenüber dem Projekt erzeugt.

Kultur Entwicklung einer Kultur für die Kollaboration, die auf gemeinsamen Werten basiert.

Sofort zu Beginn des Verbundvorhabens wurde für XENON klar, dass der Umstieg vom klassischen Email Verkehr auf Social Media basierte Kommunikationsformen große Chancen bietet, wenn sich dabei auch eine neue Kultur der Zusammenarbeit herausbildet. Ungewohnt empfanden einige Kolleginnen und Kollegen am Anfang vor allem die hohe Transparenz der Unterhaltungen in MS Teams, die von allen Teammitgliedern mitgelesen werden können. Die jungen Mitarbeiterinnen und Mitarbeiter haben diesen aus privaten WhatsApp Gruppen bekannten Stil sofort angenommen. Ältere benötigten einige Zeit, um die vollständige Information über alle laufenden Vorgänge im Team schätzen zu lernen. Grundsätzlich ist zu berichten, dass die Einführung der neuen Kommunikationsformen deutlich weniger Probleme gemacht hat, als ursprünglich vermutet. Die Kolleginnen und Kollegen arbeiten damit gern international zusammen und nutzen alle technischen Möglichkeiten von Office 365 intensiv aus. Die neue Arbeitsweise wirkt motivierend, denn gemeinsam im Team schnell voran zu kommen bei den täglichen Aufgaben, das macht Spaß. Bei XENON loben wir über Smileys. Wir geben uns Likes zur Zustimmung. Und wer es besonders gut gemacht hat, bekommt vom Team ein Herzchen geschickt. 
Zusammenarbeit/Regeln Festlegungen von Regeln der Zusammenarbeit, für die Nutzung der kollaborativen Anwendungen oder für die Dokumentation und den Austausch von Wissen.

Die modernen Kommunikationsplattformen bieten für die Teammitglieder eine Vielzahl von Nutzungsmöglichkeiten. XENON hat sich dafür entschieden, die Struktur der digitalen Plattformen durch die IT Abteilung relativ streng zu standardisieren und vorzugeben. Die Erfahrung hat gezeigt, dass für eine effiziente Zusammenarbeit gewisse Regeln notwendig sind. Es wurde deshalb ein Kultur-Kodex erarbeitet, der die wesentlichen Punkte der täglichen Kollaboration festlegt. Wie schnell muss ich antworten, wenn ich per „@“ angesprochen werde? Wie sieht die Formatierung einer MS Teams Unterhaltung aus? Wie werden wichtige Informationen mit dem Team geteilt? Wie und wo werden Dateien abgelegt? Wie benutzen wir die OneNote Notizbücher, um unsere Erkenntnisse und Festlegungen aufzuschreiben? Diese Kulturregeln wurden im Verbundvorhaben getestet, weiterentwickelt und für alle XENON Teammitglieder weltweit verbindlich eingeführt. Benannte Verantwortliche überwachen die Einhaltung der Kommunikationsregeln und stellen den Teams Vorlagen für die Strukturierung der Office 365 Apps zur Verfügung. Die Kollaboration wird zentral gelenkt, ohne dabei die Kreativität einzuschränken. Die Teams nehmen die Struktur gern an, denn so wird sichergestellt, dass die Projektarbeit effizient Fortschritte erzielt und Entscheidungen sowie deren Historie transparent nachvollziehbar sind. Die Erfahrungen mit der strukturierten digitalen Arbeitsweise sind bei XENON durchweg positiv und haben zum Gelingen der weltweiten Einführung der neuen Kommunikationsplattformen wesentlich beigetragen.

\subsubsection{Die Arbeitsgestaltung ist eine Frage von Lernprozessen und der Aushandlung zwischen Akteuren über die Zeit}

Die Erfahrungen der Partnerunternehmen dokumentieren einen intensiven individuellen und organisationalen Lernprozess bei der Gestaltung der Arbeit mit Kollaborationsplattformen über die Zeit. Dieser Lernprozess wird von stetigen Aushandlungen zwischen den unterschiedlichsten Beteiligten um die Frage geprägt, wie die Zusammenarbeit weiterentwickelt werden soll, welche Technologien eingesetzt werden und wie eine bestimmte Anwendung genutzt werden sollte. Die damit verbundenen Fragen lassen sich letztlich nur in einer Prozessperspektive beantworten: Sowohl während der Bedarfsbestimmung als auch bei der Umsetzung der Lösungen erfolgt ein ständiges Probieren, Bewerten und Weiterentwickeln.

Daher ist eine erfolgreiche Nutzung kollaborativer Anwendungen von einem ganzheitlichen Ansatz der Arbeitsgestaltung abhängig, der zugleich prozesshaft angelegt wird. Die bisher vorliegenden Erkenntnisse der sozio-technischen Systemgestaltung $[2,10,11]$ sowie des MTO-Ansatzes [12] konnten weiterentwickelt und gegenstandsbezogen konkretisiert werden. Auffällig ist heute die Notwendigkeit, auf ständige Ver- 
änderungen sehr schnell reagieren zu können. Dabei müssen die Verantwortlichen lernen, die Nutzerinnen und Nutzer angemessen am Entwicklungsprozess zu beteiligen, um deren Bedürfnisse, die durch unterschiedliche Arbeitsanforderungen und Berufsidentitäten geprägt sind, wahrzunehmen und mit den Anforderungen der Organisation und des sich rasch wandelnden Umfelds in Einklang zu bringen. In allen drei Unternehmen haben sich im Prozessverlauf größere Veränderungen gegenüber der ursprünglichen Planung ergeben. Diese Veränderungen sind sowohl durch interne Erfahrungen und Aushandlungsprozesse bedingt, als auch durch externe Impulse - wie etwa der auffallend starke Markterfolg der MS Teams Kollaborationsplattform im Jahr 2019, der alle drei Unternehmen motiviert hat, deren Nutzungsmöglichkeiten zu erproben.

Das frühere Modell von Analyse - Planung - Umsetzung bei der Einführung neuer Software ist in doppelter Hinsicht überholt. Zum einen verschmelzen die früheren drei Phasen und werden zu iterativen Entwicklungsprozessen, die sich in kurzen Zyklen wiederholen. Zum anderen ist es nicht mehr sinnvoll, von Einführung zu sprechen, weil es beim Einsatz von Informations- und Kommunikationslösungen eher um Weiterentwicklungen vorhandener Lösungen geht.

Für die aktive Aneignung von Kollaborationsplattformen ist es entscheidend, dass die Nutzerinnen und Nutzer durch die Nutzung eine substanzielle Unterstützung bei ihrer täglichen Arbeit erfahren und selbst einen Sinn in dem durch die Plattform ermöglichten Austausch mit anderen Beschäftigten sehen. Mehr Autonomie, mehr Transparenz und mehr Partizipation zu erreichen, sind vielfach artikulierte Bedürfnisse der Beschäftigten, die sie zur Nutzung der neuen technischen Werkzeuge motivieren. Entsprechend erweist sich die Kontroll-Thematik als kritischer Punkt für die Nutzung: Gewinnen die Beschäftigten den Eindruck, dass der Einsatz von Kollaborationsplattformen einer erweiterten Management-Kontrolle dienen soll, verlieren die neuen Tools spürbar an Attraktivität. Ein effektiver Einsatz von Kollaborationsplattformen ist jedoch davon abhängig, dass Inhalte und Wissen aktiv geteilt werden, dies setzt eine intrinsische Motivation der Beschäftigten voraus.

Für eine Arbeitsgestaltung von Kollaborationsplattformen reicht ein enger Bezug auf ein Team als Ansatzpunkt nicht mehr aus. Die Arbeitsgestaltung muss stattdessen der Tatsache Rechnung tragen, dass Kollaborationsplattformen i. d. R. für die Zusammenarbeit im gesamten Unternehmen eingesetzt werden, d. h. quer zu bestehenden organisationalen Grenzen wie etwa Bereichen Vernetzung in Arbeitsgruppen ermöglichen können bzw. sollen, die den Wissensaustausch und die Kommunikation fördern. Dies fordert die bestehenden Praktiken und die Unternehmenskultur vielfach heraus. 


\subsection{Forschungslücken, Ausblick auf möglicherweise fortlaufende Forschungsarbeit}

Im Projekt wurde die Zusammenarbeit im Team und Projekt untersucht und neue Betreuungsrollen deutlich gemacht. Doch was bedeutet für Führungskräfte die Arbeit mit Kollaborationsplattformen, die mit den Vernetzungsmöglichkeiten quer zu organisationalen Strukturen, die bestehenden Hierarchien infrage stellt? Insbesondere die Anforderungen an das mittlere Management sind hier interessant zu untersuchen: wie führen, sodass das Team seine Arbeit gut bewältigen kann? Für was ist die Führungskraft zuständig? Was bedeutet es für die Führungskraft, wenn die Kommunikation für alle im Team transparent ist?

Außerdem ist der Blick vermehrt auf die Auswirkungen der verstärkten Zusammenarbeit über Plattformen auf den sozialen Zusammenhalt, die soziale Nähe sowie die Integration der Mitarbeiterinnen und Mitarbeiter im gesamten Betrieb zu untersuchen. Was bedeuten die digitalen Werkzeuge und die räumlich und zeitlich entkoppelte Arbeit für soziale Beziehungen im Betrieb? Verflüssigen sich die Teamstrukturen? Inwieweit werden durch Zusammenarbeit z. B. mit Kunden auch Organisationsgrenzen aufgeweicht und mit welchen Konsequenzen?

Darüber hinaus werfen die Projekterfahrungen die Frage auf, wie die Unternehmen in der digitalen Transformation die Normen einer human-orientierten Arbeitsgestaltung umsetzen können. Die Aufgaben der Arbeitsgestaltung sind vielfältig, komplex und auf viele Köpfe verteilt, d. h. unterschiedliche Teilexpertisen (IT, HR, Arbeitsschutz, Fachverantwortlichkeit) müssen zusammengebracht werden. Viele Aspekte sind in Beteiligungsprozessen mit Beschäftigten oder Betriebs- und Personalräten zu verhandeln. Es ist bislang nicht ausgeleuchtet worden, wie die Unternehmen die notwendige sozio-technische Kompetenz zur human- und prozessorientierten Arbeitsgestaltung aufbauen und die Zusammenarbeit entsprechend den Bedürfnissen der Nutzerinnen und Nutzer organisieren können.

\subsection{Hinweis auf Transfermaterialien}

Für Menschen, die sich in Ihrem Unternehmen für eine menschenorientierte Gestaltung kollaborativer Arbeit einsetzen oder als Externe an der Arbeitsgestaltung in einem Unternehmen beteiligen, hat das Projekt Materialien erarbeitet, die auf collaboteam.de öffentlich zugänglich sind: 1) „Arbeit mit Kollaborationsplattformen“ - eine Broschüre mit Gestaltungsempfehlungen 2) Ein open access Buch mit praxisorientierter Aufarbeitung der Projektergebnisse ,Eine neue Qualität der Zusammenarbeit im Unternehmen - Die Arbeit mit Kollaborationsplattformen gestalten“. 


\section{Projektpartner und Aufgaben}

- Georg-August-Universität, Kooperationsstelle Hochschulen und Gewerkschaften

Entwicklung integrierter humaner Konzepte der soziotechnischen Gestaltung verteilten Arbeitens mit kollaborativer Software und von PE/OE Verfahren

- Soziologisches Forschungsinstitut Göttingen e. V.

Entwicklung von Gestaltungsempfehlungen für die humane Nutzung kollaborativer Software in der Team- und Projektarbeit und Bestandsaufnahme der Gestaltungsanforderungen von Kollaboration in KMU

- Carl Zeiss Digital Innovation GmbH (ehem. Saxonia Systems AG)

Entwicklung von Kollaborationslösungen für die räumlich verteilte, agile Softwareentwicklung

- Xenon Automatisierungstechnik GmbH

Erprobung von Social Media Anwendungen zur Workflow-Optimierung im Anlagenbau

- GIS Gesellschaft für Informationssysteme AG

Erprobung von Office 365 Cloud Lösungen für mobile Büroarbeit in der IT Dienstleistung

\section{Literatur}

1. Williams SP, Schubert P (2015) Social Business Readiness Studie 2014. CEIR Research Report, 01/2015, Universität Koblenz-Landau

2. Mohr BJ, van Amelsvoort P (Hrsg) (2016) Co-creating humane and innovative organizations. Evolutions in the practice of socio-technical system design, Global STS-D Network, Portland, $\mathrm{ME}$

3. Greeven CS, Williams SP (2017) Enterprise collaboration systems: adressing adoption challenges and the shaping of socialtechnical systems. Int J Inf Syst Proj Manage 5(1):5-23

4. McAfee A (2009) Enterprise 2.0. New collaborative tools for your organization's toughest challenges. Harvard Business Press, Boston, Mass

5. Hardwig T, Klötzer S, Boos M (2020) Software-supported collaboration in small and mediumsized enterprises. Meas Bus Excell 24(1):1-23

6. Paul G (2018) Die Befragung von KMUs zur Kollaborativen Team- und Projektarbeit. www. collaboteam.de

7. Hardwig T, Klötzer S, Boos M (2019) The benefits of software-supported collaboration for small and medium sized enterprises. A literature review of empirical research papers. In: IFKAD (Hrsg) Proceedings. Knowledge ecosystems and growth. 14th International Forum on Knowledge Asset Dynamics. Arts for Business Institute, University of Basilicata, Basilicata, S $1024-1034$

8. Ulich E (2011) Arbeitspsychologie, 7., neu überarb. und erw. Aufl. vdf Hochschulverl. an der ETH, Zürich

9. Klötzer S, Hardwig T, Boos M (2017) Gestaltung internetbasierter kollaborativer Team- und Projektarbeit. Gruppe. Interaktion. Organisation. Zeitschrift für Angewandte Organisationspsychologie 31(5):133. https://doi.org/10.1007/s11612-017-0385-3 
10. Sydow J (1985) Der soziotechnische Ansatz der Arbeits- und Organisationsgestaltung. Darstellung, Kritik, Weiterentwicklung. Campus Forschung, Band 428. Campus-Verl., Frankfurt a.M.

11. Clegg CW (2000) Sociotechnical principles for system design. Appl Ergon 31(5):463-477. https://doi.org/10.1016/S0003-6870(00)00009-0

12. Strohm O, Ulich E (1997) Unternehmen arbeitspsychologisch bewerten. Ein Mehr-EbenenAnsatz unter besonderer Berücksichtigung von Mensch, Technik, Organisation. Zeitschrift für Arbeitswissenschaft 51(1):11-19s

Open Access Dieses Kapitel wird unter der Creative Commons Namensnennung 4.0 International Lizenz (http://creativecommons.org/licenses/by/4.0/deed.de) veröffentlicht, welche die Nutzung, Vervielfältigung, Bearbeitung, Verbreitung und Wiedergabe in jeglichem Medium und Format erlaubt, sofern Sie den/die ursprünglichen Autor(en) und die Quelle ordnungsgemäß nennen, einen Link zur Creative Commons Lizenz beifügen und angeben, ob Änderungen vorgenommen wurden.

Die in diesem Kapitel enthaltenen Bilder und sonstiges Drittmaterial unterliegen ebenfalls der genannten Creative Commons Lizenz, sofern sich aus der Abbildungslegende nichts anderes ergibt. Sofern das betreffende Material nicht unter der genannten Creative Commons Lizenz steht und die betreffende Handlung nicht nach gesetzlichen Vorschriften erlaubt ist, ist für die oben aufgeführten Weiterverwendungen des Materials die Einwilligung des jeweiligen Rechteinhabers einzuholen.

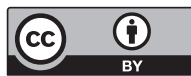

